\title{
Detuned-resonator induced transparency in dielectric-loaded plasmonic waveguides
}

\author{
Zhanghua Han, ${ }^{1,2, *}$ Cesar E. Garcia-Ortiz, ${ }^{1,3}$ Ilya P. Radko, ${ }^{1}$ and Sergey I. Bozhevolnyi ${ }^{1}$ \\ ${ }^{1}$ Department of Technology and Innovation, University of Southern Denmark, Niels Bohrs Alle 1, Odense M DK-5230, Denmark \\ ${ }^{2}$ Center for THz Research, China Jiliang University, Hangzhou 310018, China \\ ${ }^{3}$ UANL, Facultad de Ciencias Físico-Matemáticas, San Nicolás de los Garza, Nuevo León 66450, Mexico \\ *Corresponding author: zhh@iti.sdu.dk
}

Received December 5, 2012; accepted January 20, 2013;

posted January 23, 2013 (Doc. ID 181224); published March 12, 2013

\begin{abstract}
We report on the experimental demonstration of detuned-resonator induced transparency in the near-infrared ( 800 nm) using two detuned racetrack resonators side-coupled to a bus waveguide. Both resonators and the bus waveguide are in the form of dielectric-loaded surface plasmon polariton waveguides. Leakage radiation microscopy imaging is employed to measure transmission spectra, featuring local maxima at intermediate wavelengths with asymmetrical profiles that are found in good agreement with full-wave numerical simulation results. (c) 2013 Optical Society of America OCIS codes: $240.6680,230.5750$.
\end{abstract}

The use of surface plasmon polaritons (SPPs) to guide electromagnetic radiation offers the advantages of better confinement and the possibility of hybrid integration between electronics and photonics, compared to the conventional optical waveguides; thus the use of SPPs has been intensively investigated in recent years [1]. Among various plasmonic waveguides proposed to date, dielectric-loaded SPP waveguides (DLSPPWs) [2] are of special interest in terms of fabrication simplicity and the tradeoff between mode confinement and propagation length. Many passive optical elements based on DLSPPWs, including bends and directional couplers [3], waveguide ring resonators [4], and Bragg gratings [4] ], have been experimentally demonstrated. Active control of SPP propagation in the form of modulation [5] or switching [6] has also been realized using the thermooptical effect of polymers. These developments demonstrate the versatility of the DLSPPW technology platform and its suitability for plasmon-based nanophotonics.

In this Letter we report on the experimental demonstration of a plasmonic analogue to the classical electromagnetically induced transparency (EIT) phenomenon in the near-infrared regime using DLSPPW resonators. This analogue, known as DRIT, is due to the destructive interference between optical waves coupled back to the output bus waveguide after different optical pathways in the resonators. DRIT has been well investigated both theoretically and experimentally using coupled photonic resonators $[\underline{7}, \underline{8}]$ or photonic-crystal cavities. There are also some theoretical explorations of the plasmonic analogue of EIT using plasmonic resonators in the literature $[9,10]$. Plasmon-induced transparency $[\underline{11]}$ has been experimentally demonstrated in metamaterials [12]. In principle, the EIT effect resulting from the coupling between two different radiative states in metamaterials is equivalent to DRIT in that there are also two optical pathways, one from the bright state directly to the free space and the other through the dark state. EIT in optical metamaterials is also a result of the plasmonic effect; however, it is due to the localized surface plasmon resonances where the metallic part of the metamaterials can be regarded as electrical dipoles [13]. To date there are no experimental results of the DRIT in integrated plasmonics using plasmon-based waveguide resonators.

Our proposed and investigated structure is based on DLSPPWs and consists of two racetrack resonators evanescently coupled to a central bus waveguide (Fig. 1). Both resonators and the bus waveguide represent DLSPPWs composed of poly(methyl methacrylate) (PMMA) stripes with the same width $W$ of $320 \mathrm{~nm}$ and height $H$ of $300 \mathrm{~nm}$ residing on a $70 \mathrm{~nm}$ thickness gold film evaporated on a silica substrate. The dimensions of the PMMA stripes are chosen intentionally to ensure single-mode operation. The cross section of the DLSPPWs is shown as the inset in Fig. 1(a). Considering that the roundtrip loss inside the resonator is quite high, resulting from both bending loss and propagation loss, racetrack resonators are used to enhance the coupling between the resonators and the bus waveguide to achieve a high transmission contrast between resonant and nonresonant wavelengths. In this Letter the gap $g$ between two resonators and the bus waveguide is $100 \mathrm{~nm}$ and the length $L$ of the straight section in the two racetrack resonators is kept as $700 \mathrm{~nm}$. We keep the radius of one resonator at $R_{1}=2.0 \mu \mathrm{m}$ and slightly change that of the second resonator $R_{2}$ with the detuning denoted as $d R$. Since destructive interference between optical waves after different optical pathways is expected to realize the intermediate transmission peaks, we can estimate the value of $d R$ by solving the equation $n_{\text {eff }} k_{0} \bullet 2 \pi d R=\pi$, where $n_{\text {eff }}$ is the mode effective index

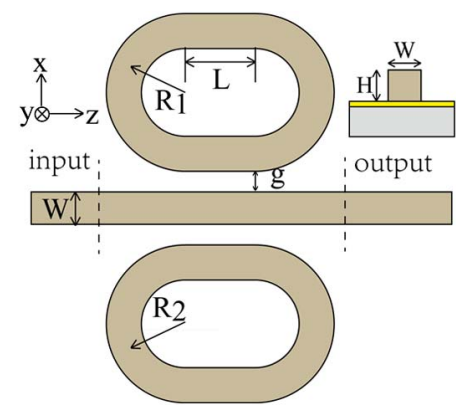

Fig. 1. (Color online) Top view schematic of two detuned resonators with radii $R_{1}$ and $R_{2}$ side-coupled to a bus waveguide. Inset is the cross section of the DLSPPWs. 
and $k_{0}$ is the free-space wave number. We can find $d R$ is on the order of tens of nanometers for the near-infrared wavelengths.

A similar type of structure based on optical ring resonators has been numerically studied previously [8] using the transfer matrix method. However, the interaction between two rings was ignored [8] to simplify the analysis, which, we find, is not proper to predict the transmission behavior. So, in this Letter, we use a full-wave numerical technique to simulate the structure. The finite element method-based commercial software of Comsol Multiphysics is employed, in which the tabulated experimental data of complex refractive index for Au [14] are used. In the simulations, scattering boundary conditions are used all around the structure except the output waveguide port where uniaxial perfectly matched layers are used to minimize the reflection. The incident light is from the left PMMA end, and the transmission is defined as the integration of Poynting vector component $\mathrm{Pz}$ within the PMMA stripe across the output plane normalized to that across the input plane when there is only the reference straight waveguide present. We scanned the wavelength and thus got the transmission spectrum.

We first investigate the case when there is only one racetrack resonator coupled to the bus waveguide. The two dashed curves shown in Fig. 2(a) represent the transmission spectra when the radius is equal to 2.0 and $2.04 \mu \mathrm{m}$, with the transmission minima around 809 and $819 \mathrm{~nm}$, respectively. When both resonators are present, one can see in the solid blue curve that an extra transmission peak at the intermediate wavelength shows up, however, with an asymmetrical profile with respect to the original two transmission minima. Although the transmission peak is at the wavelength where the two dashed curves intersect, the transmission spectrum for the DRIT is not a superposition of the two spectra with only one resonator. This suggests that the asymmetrical profile is a result of the interactions between the two resonators.
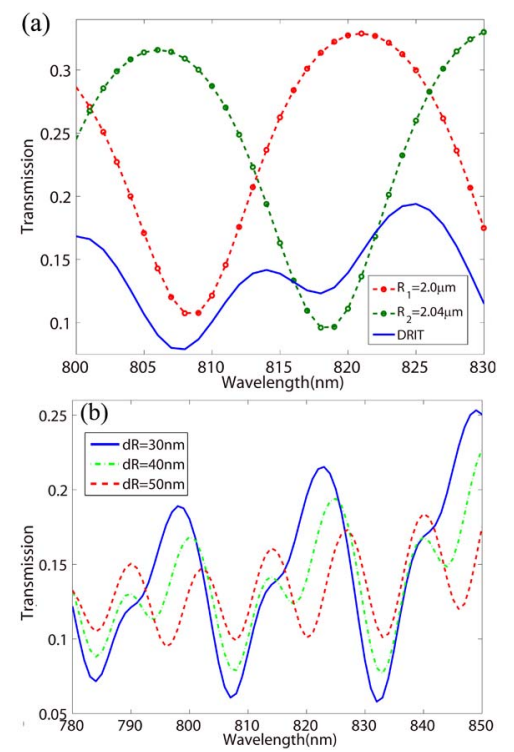

Fig. 2. (Color online) Numerically calculated transmission spectra for different structures. (a) Dashed curves, only one resonator; solid curve, two detuned resonators. (b) DRIT with different radius detuning.
This behavior, has also been found in the optical transparency due to the coupling between detuned electrical dipoles [13]. The asymmetric coupling conditions between the bus waveguide and the two resonators as well as the different roundtrip losses in the two racetrack resonators may also contribute to this asymmetric behavior. Figure 2(b) presents the calculated transmission spectra when radius $R_{1}$ is kept as $2.0 \mu \mathrm{m}$ while $R_{2}$ is changed with different detuning. For all the results, one can see similar EIT-like transmission spectra with asymmetrical profiles as shown in Fig. 2(a). As the detuning $d R$ is larger, the transmission peak also becomes more pronounced.

We fabricated the depicted double-resonator structure using electron beam lithography (EBL). After thermally evaporating a $70 \mathrm{~nm}$ thick gold layer to the silica substrate and spin coating a $300 \mathrm{~nm}$ thick PMMA (950 PMMA A4 from Micro Chem), an electron beam exposure was used to pattern the PMMA, and after developing, the structure was obtained. Atomic force microscopy was used to measure the structure, and by adjusting the dose of the EBL exposure we made sure the width of the PMMA stripes was $320 \mathrm{~nm}$ and $g$ was around $100 \mathrm{~nm}$. Figure 3(a) shows an optical microscopy image of one of the fabricated structures, in which one can see clearly the PMMA stripes in the form of two racetrack resonators side-coupled to the central waveguide. The straight bus waveguide starts from the left with a tapered structure whose width decreases from $4 \mu \mathrm{m}$ to $320 \mathrm{~nm}$ over a length of $10 \mu \mathrm{m}$. This taper is used to couple light efficiently into the SPP mode by directly illuminating the tapered region with a moderately focused laser beam. The slow variation of the taper width will ensure the excited mode in the taper will experience low reflection when it is coupled into the bus waveguide.

A tunable Ti:sapphire laser $(\sim 10 \mathrm{~mW})$ is used as the illumination source and the polarization is set along the bus waveguide to excite the DLSPPW mode. The leakage radiation microscopy (LRM) setup with a high-numericalaperture objective placed below the silica substrate to collect the leakage radiation signal from the DLSPPW mode through the glass slide is used to map the field of the SPP propagating in the detuned-resonator structure. Figure 3(b) shows a typical LRM image when the structure with a $d R$ equal to $40 \mathrm{~nm}$ is illuminated at the wavelength of $819 \mathrm{~nm}$. Since the SPP field in the taper area is quite strong compared to that in the double-resonator structure, which is of more interest in this Letter, the former SPP field has been blocked in Fig. 3(b) to highlight the SPP propagation in the double resonator. Considering the fact that the DLSPPW mode index is larger (around 1.3) than that (around 1) of the SPP propagating along the $\mathrm{Au} /$ air interface, in the LRM measurements, a low-pass filter in the Fourier plane is used to minimize

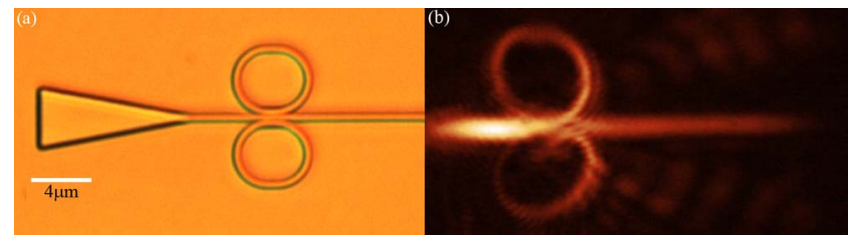

Fig. 3. (Color online) (a) Optical microscopy image of fabricated structures. (b) LRM image of the SPP field for $d R=40 \mathrm{~nm}$ for the wavelengths of $819 \mathrm{~nm}$. 
the influence of the SPP at the Au/air interface in the obtained LRM images. In Fig. 3(b) the SPP fields before and after the double-resonator structure are used to calculate the transmission. After tuning the incident wavelength, we can obtain LRM images at different wavelengths, with which the transmission spectrum is obtained.

The transmission spectra obtained in this manner from the experimental LRM images for the two doubleresonator structures where $R_{1}$ is kept as $2.0 \mu \mathrm{m}$ while $R_{2}=2.04$ or $2.05 \mu \mathrm{m}$ are shown in Fig. 4 . Comparing these experimental results to those numerical results from Comsol shown in Fig. 2(b), one can see that quite good agreements between the two results have been achieved. The induced transmission peaks have the same asymmetry in both results, and they are also more pronounced when the radius detuning becomes larger. The number of transmission minima and peaks, the order of magnitudes of the transmission, are also quite consistent. One can also notice that the experimental result in Fig. 4(a) for $d R=40 \mathrm{~nm}$ is more like the numerical result for $d \overrightarrow{R=}$ $30 \mathrm{~nm}$ shown as the blue solid curve in Fig. 2(b). The same trend is also found for $d R=50 \mathrm{~nm}$ in Fig. 4(b). We attribute the discrepancy mainly to the difference between the PMMA refractive index of 1.484 used in the numerical simulations and a smaller value of it in the real experiment. This viewpoint is supported with the evidences that all transmission minima in Fig. 4 are blue shifted by $\sim 3 \mathrm{~nm}$ as compared to the corresponding numerical results in Fig. 2(b) and that in Fig. 4 the free spectral range is a little bit larger than in the calculated results.

Having realized the EIT-like transmission spectrum using the plasmonic detuned resonator, we can further evaluate the slow-light effect that can be achieved using this DRIT. The light group index can be calculated using the equation $n_{g}=n_{p}-\lambda d n_{p} / d \lambda$, where $n_{g}$ and $n_{p}$ are the group index and phase index at the wavelength of $\lambda$ in the waveguide, respectively. Using the results from numerical simulations, we find the calculated at the transmission peak around the wavelength of $812 \mathrm{~nm}$ is about 2.6 (2.8) for the radius detuning of $40 \mathrm{~nm}(50 \mathrm{~nm})$. The bandwidth-delay product (BDP) is calculated to be $7.5 \%$

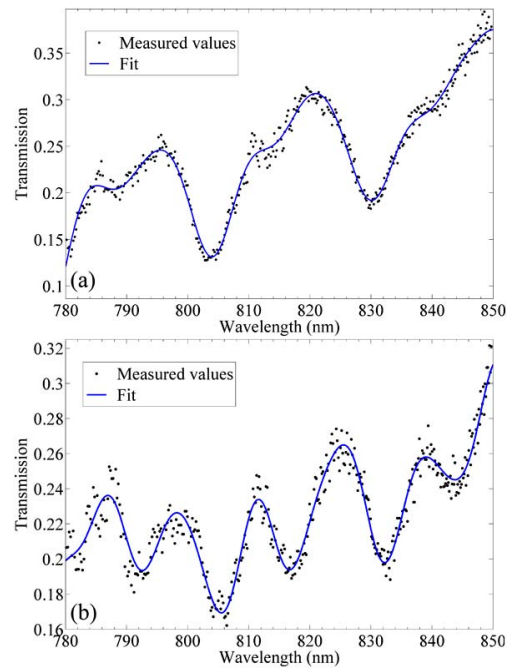

Fig. 4. (Color online) Transmission spectra from LRM for double-resonator structures with the radius detuning $d R$ being $40 \mathrm{~nm}$ in (a) and $50 \mathrm{~nm}$ in (b). for the case when $d R$ equals $50 \mathrm{~nm}$. The relatively low values of group index and BDP are mainly attributed to the roundtrip loss in the DLSPP racetrack resonators. We expect that the group index can be improved by further optimizing the length of the straight section in the two racetrack resonators to increase the contrast in the transmission spectra.

In a summary, we have fabricated the double-resonator structures based on DLSPPWs using EBL and experimentally demonstrated the phenomenon of DRIT in these structures using the LRM technique. These results, although obtained in the near infrared, can be extended to other wavelengths. Numerical simulations have also been performed to understand the phenomenon and to help estimate the group index achieved in the fabricated double-resonator structures. Although the achieved group index is not high due to plasmonic resonator loss, the DRIT still provides a new means of waveguide dispersion control and we also expect that DRIT can find broad applications in the on-chip sensing area, e.g., in temperature sensing [15], because the induced transmission peaks in DRIT are more sensitive to index change.

This work was supported by the Danish Council for Independent Research (Contract No. 09-072949 ANAP). Z. Han also acknowledges support from the National Natural Science Foundation of China (No. 61107042). C. E. Garcia-Ortiz gratefully acknowledges the support from CONACYT scholarship 228959 and Facultad de Ciencias Físico-Matemáticas.

\section{References}

1. D. K. Gramotnev and S. I. Bozhevolnyi, Nat. Photonics 4, 83 (2010).

2. S. Massenot, J. Grandidier, A. Bouhelier, G. Colas des Francs, L. Markey, J.-C. Weeber, A. Dereux, J. Renger, M. U. Gonzàlez, and R. Quidant, Appl. Phys. Lett. 91, 243102 (2007).

3. Z. Chen, T. Holmgaard, S. I. Bozhevolnyi, A. V. Krasavin, A. V. Zayats, L. Markey, and A. Dereux, Opt. Lett. 34, 310 (2009).

4. T. Holmgaard, Z. Chen, S. I. Bozhevolnyi, L. Markey, A. Dereux, A. V. Krasavin, and A. V. Zayats, Appl. Phys. Lett 94, 051111 (2009).

5. J. Gosciniak, S. I. Bozhevolnyi, T. B. Andersen, V. S. Volkov, J. Kjelstrup-Hansen, L. Markey, and A. Dereux, Opt. Express 18, 1207 (2010).

6. K. Hassan, J.-C. Weeber, L. Markey, A. Dereux, A. Pitilakis, O. Tsilipakos, and E. E. Kriezis, Appl. Phys. Lett. 99, 241110 (2011).

7. D. Smith, H. Chang, K. Fuller, A. Rosenberger, and R. Boyd, Phys. Rev. A 69, 063804 (2004).

8. Y. Zhang, S. Darmawan, L. Y. M. Tobing, T. Mei, and D. H. Zhang, J. Opt. Soc. Am. B 28, 28 (2011).

9. R. Kekatpure, E. Barnard, W. Cai, and M. Brongersma, Phys. Rev. Lett. 104, 243902 (2010).

10. Z. Han and S. I. Bozhevolnyi, Opt. Express 19, 3251 (2011).

11. S. Zhang, D. A. Genov, Y. Wang, M. Liu, and X. Zhang, Phys. Rev. Lett. 101, 047401 (2008).

12. N. Liu, T. Weiss, M. Mesch, L. Langguth, U. Eigenthaler, M. Hirscher, C. Sönnichsen, and H. Giessen, Nano Lett. 10, 1103 (2010).

13. A. B. Evlyukhin, S. I. Bozhevolnyi, A. Pors, M. G. Nielsen, I. P. Radko, M. Willatzen, and O. Albrektsen, Nano Lett. 10, 4571 (2010).

14. P. B. Johnson and R. W. Christy, Phys. Rev. B 6, 4370 (1972).

15. T. B. Andersen, S. I. Bozhevolnyi, L. Markey, and A. Dereux, Opt. Express 19, 26423 (2011). 